\title{
Perianal Endometriosis Mimicking Recurrent Perianal Abscess
}

\author{
Mohd lqbal $^{a}$ Vijay Thumbe ${ }^{b}$ Ravish Dhange \\ Sui Yum Chan ${ }^{b}$ Satish Bhalerao ${ }^{b}$ \\ ${ }^{a} Q u e e n ' s$ Hospital, Burton, and bepartment of Surgery and Pathology, City \\ Hospital, Birmingham, UK
}

\section{Key Words}

Perianal endometriosis - Recurrent perianal abscess

\begin{abstract}
Endometriosis of the perianal region is an extremely rare condition usually seen in episiotomy scars and can involve the septum separating vagina and anal canal. The clinician is unlikely to suspect it if there is no scar in the perineum and patient the does not give a history of episiotomy. Moreover it is difficult to suspect when the patient does not report cyclical pain and therefore it is likely to be treated as perianal sepsis. We report the rare case of a 35-year-old woman who presented with recurrent painful nodule of endometriosis of the perianal area without previous episiotomy.
\end{abstract}

\section{Introduction}

Endometriosis is a clinical entity characterised by the presence of functional endometrial tissue in an anatomical location outside the uterine cavity. This condition is sometimes seen in the scars of caesarean section where it can be confused with incisional hernia [1]. It is rarely ( $0.2 \%$ of ectopic endometrium) seen in the perineum where it is usually localised in episiotomy scars [2]. Very rarely it can be seen in the perianal area [3]. The characteristic cyclical pain is uncommon [1].

\section{Case Report}

A 35-year-old lady presented as an emergency to different hospitals on three occasions with a history of severe pain in the perianal area. There was no change in the severity of symptoms in relation to the menstrual cycle during the first two episodes. Subsequently she started noticing the symptoms coinciding with the periods. She had had two deliveries, the first one a normal delivery without episiotomy and the second one caesarean section. 
At the time of first consultation she was noted to have a $2 \mathrm{~cm}$ tender nodule in the perianal area at the 2 o'clock position which was thought to be a perianal abscess. This was incised and drained and as no pus was seen, she was treated as suffering from cellulitis. She presented with similar symptoms a few months later to a different hospital and was treated for recurrent perianal abscess. During her third presentation some blood clots were seen in the nodule and hence an excision biopsy was done which showed endometrial tissue (fig. 1). Her white blood cell count was normal and C-reactive protein was mildly elevated. Subsequently she had recurrence of symptoms after nine months which was treated symptomatically. She was further investigated with flexible sigmoidoscopy and an MRI scan of the pelvis and perineum which did not show any pelvic endometriosis. She was referred to a gynaecologist for hormonal treatment as nothing was shown on MRI scan.

\section{Discussion}

Perianal endometriosis is very rare. A PubMed search showed only twelve cases in eight different case reports. It is relatively more common in episiotomy scars where endometrial tissue gets implanted [2]. It is rarely suspected by the clinician as a differential diagnosis of perianal problems, and the characteristic cyclical pain is uncommon in endometriosis [1]. As perianal sepsis is more commonly dealt with by junior surgeons, unusual presenting features of this condition may not be recognised. This may lead to inappropriate diagnosis of perianal sepsis and unnecessary operations. A high degree of suspicion is the key to diagnosis, particularly when a patient presents with recurrent perianal pain and no pus is found. Fine needle aspiration cytology has been shown to be helpful in diagnosis [4]. Anal endosonography can help to know the precise anatomical location and involvement of the external anal sphincter [5]. Involvement of senior surgeons and biopsy of unusual lesions in patients presenting with symptoms of recurrent perianal pain would establish the diagnosis early. Wide local excision sparing anal sphincter cures the condition and hormonal treatment can be used when it involves multiple sites [2] or when excision is not possible.

\section{Conclusion}

Painful perianal lesions/swellings raise the suspicion of perianal abscess. In cases of recurrence of such lesions and fistulation, Crohn's disease is often suspected. In females such lesions can be endometriosis, particularly when no pus is found or the problem is cyclical. A high degree of suspicion and biopsy are helpful in diagnosis. 
Fig. 1. Endometrial glands with typical stroma surrounded by fibrosis. Haemosiderin deposit is seen in the surrounding tissue.

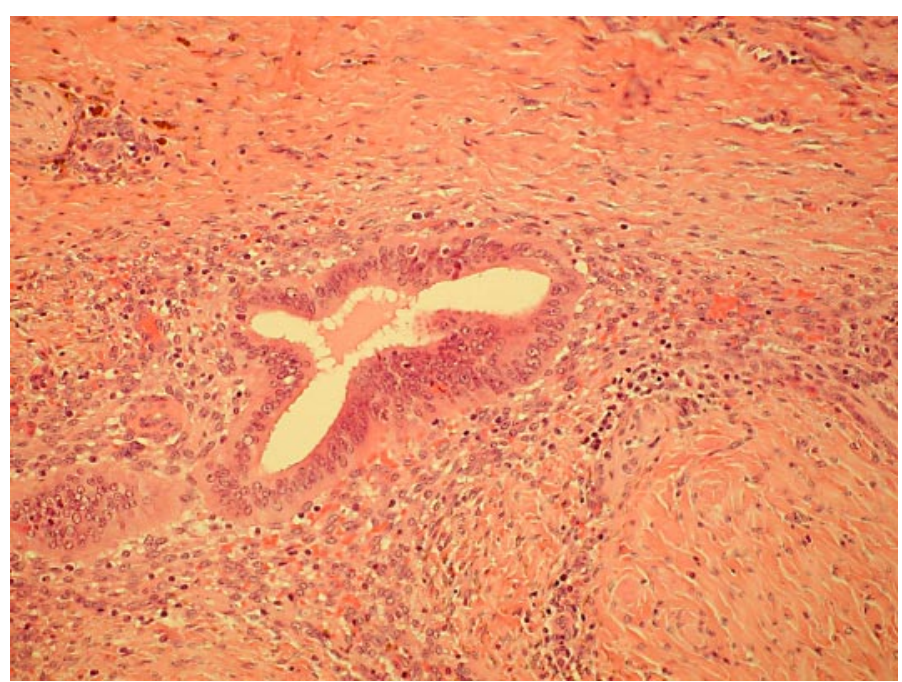




\section{References}

1 Rao A, Devalia H, Zaidi A: Post-caesarean incisional hernia or scar endometrioma? Surgeon 2006;4:55-56.

-2 Marquez J, Marquez JC, Arraztoa JA, Perez G, Espinoza A: Extrapelvic endometriosis involving the perineum. Rev Chil Obstet Ginecol 1995;60:1-4.

-3 Schweiger W, Bacher H, Cerwenka H, Mischinger HJ: Perianal endometriosis with involvement of the external anal sphincter. Eur J Surg 1999;165:615-617.

4 Simsir A, Thorner K, Waisman J, Cangiarella J: Endometriosis of abdominal scars: a report of three cases diagnosed by fine needle aspiration biopsy. Am Surg 2001;67:984-986.

-5 Watanabe M, Kamiyama G, Uamazaki K, Hiratsuka K, Takata M, Tsunoda A, Shibusawa M, Kusano M: Anal endosonography in the diagnosis and management of perianal endometriosis: report of a case. Surg Today 2003;33:630632. 